OPEN ACCESS

Edited by: Wolfgang R. Streit,

University of Hamburg, Germany

Reviewed by:

Dinesh Sriramulu,

Shres Consultancy (Life Sciences),

India

Florian-Alexander Herbst, Aalborg University, Denmark

${ }^{*}$ Correspondence: Youngjoo Byun yjbyun1@korea.ac.kr

Hee-Deung Park

heedeung@korea.ac.kr

Specialty section: This article was submitted to Systems Microbiology, a section of the journal Frontiers in Microbiology

Received: 22 February 2017 Accepted: 15 May 2017

Published: 30 May 2017

Citation:

Kim T-S, Ham S-Y, Park BB, Byun Y and Park H-D (2017) Lauroyl Arginate

Ethyl Blocks the Iron Signals Necessary for Pseudomonas aeruginosa Biofilm Development.

Front. Microbiol. 8:970 doi: 10.3389/fmicb.2017.00970

\section{Lauroyl Arginate Ethyl Blocks the Iron Signals Necessary for Pseudomonas aeruginosa Biofilm Development}

\author{
Taek-Seung Kim ${ }^{1}$, So-Young Ham ${ }^{1}$, Bernie B. Park², Youngjoo Byun ${ }^{2 *}$ and \\ Hee-Deung Park ${ }^{1 *}$ \\ ${ }^{1}$ School of Civil, Environmental and Architectural Engineering, Korea University, Seoul, South Korea, ${ }^{2}$ College of Pharmacy, \\ Korea University, Sejong, South Korea
}

Pseudomonas aeruginosa is a ubiquitous gram-negative bacterium capable of forming a biofilm on living and non-living surfaces, which frequently leads to undesirable consequences. We found that lauroyl arginate ethyl (LAE), a synthetic non-oxidizing biocide, inhibited biofilm formation by $P$. aeruginosa at a sub-growth inhibitory concentration under both static and flow conditions. A global transcriptome analysis was conducted using a gene chip microarray to identify the genes targeted by LAE. In response to LAE treatment, $P$. aeruginosa cells up-regulated iron acquisition and signaling genes and down-regulated iron storage genes. LAE demonstrated the capacity to chelate iron in an experiment in which free LAE molecules were measured by increasing the ratio of iron to LAE. Furthermore, compared to untreated cells, $P$. aeruginosa cells treated with LAE exhibited enhanced twitching motility, a phenotype that is usually evident when the cells are starved for iron. Taken together, these results imply that LAE generated iron-limiting conditions, and in turn, blocked iron signals necessary for $P$. aeruginosa biofilm development. As destroying or blocking signals leading to biofilm development would be an efficient way to mitigate problematic biofilms, our findings suggest that $L A E$ can aid in reducing $P$. aeruginosa biofilms for therapeutic and industrial purposes.

\section{Keywords: biofilm, biofilm inhibitor, iron, lauroyl arginate ethyl, Pseudomonas aeruginosa}

\section{INTRODUCTION}

Pseudomonas aeruginosa is an opportunistic pathogen of various plants and animals (Stover et al., 2000; He et al., 2004). In humans, $P$. aeruginosa frequently causes serious infections in the lung airways, the urinary tract, of burns, etc., especially in immunocompromised patients (Driscoll et al., 2007). In addition, P. aeruginosa can contaminate medical devices such as catheters and joint 
prostheses, which can lead to serious medical complications (Weinstein and Darouiche, 2001). However, it is difficult to appropriately treat these infections or contamination by $P$. aeruginosa (Percival et al., 2015), mainly because $P$. aeruginosa can form a biofilm on both inert and living surfaces (Costerton et al., 1999). A biofilm is a surface-attached microbial community embedded in a self-produced hydrated polymeric matrix. Because the diffusion of antibiotics or biocides into cells across the polymeric matrix is retarded by a biofilm and biofilm cells grow slowly, biofilm cells are more resistant to antimicrobial agents than the corresponding planktonic cells (Costerton et al., 1999).

In a manner, similar to that exhibited in other bacteria, biofilm development in $P$. aeruginosa occurs in consecutive stages: initial attachment, microcolony formation, maturation into a differentiated biofilm, and dispersal of the planktonic cells. Studies over the past two decades have identified various stage-by-stage signals or cues that trigger biofilm development at the molecular level. The signal molecules leading to biofilm development that have been most frequently studied are autoinducers (e.g., 3-oxododecanoyl homoserine lactone), which are produced in response to the local population density (Davies et al., 1998). Autoinducers are essential for the differentiation of $P$. aeruginosa into a mushroom-like mature biofilm structure (Davies et al., 1998). Cyclic diguanylate (c-di-GMP) is a secondary metabolite that is synthesized intracellularly by various bacteria including $P$. aeruginosa, and it affects the various stages of $P$. aeruginosa biofilm development. High levels of cellular c-di-GMP facilitate biofilm formation, while low levels increase the dispersal of planktonic cells from a mature biofilm (Borlee et al., 2010). Intracellular iron has also been reported to serve as a signal for $P$. aeruginosa in the initial attachment (O'Toole and Kolter, 1998a), microcolony formation (Singh et al., 2002), and maturation into a differentiated biofilm (Banin et al., 2005). In addition, cis-decenoic acids, 4-quinolones, and diketopiperazines have been reported to be signal molecules for biofilm development in $P$. aeruginosa (Jimenez et al., 2012).

Destroying or blocking the signal molecules involved in biofilm development is a promising strategy for mitigating noxious $P$. aeruginosa biofilms. This approach has the advantage of reducing the appearance of resistant strains in response to antibiotic administration (Hentzer et al., 2003). Various approaches have been suggested for this strategy, such as the introduction of enzymes that degrade the autoinducers [e.g., acylase and lactonase (Dong and Zhang, 2005)], molecules that block the binding of the autoinducers to their receptors [e.g., furanone C-30 (Hentzer et al., 2002) and 6-gingerol (Kim et al., 2015)], inhibitors of the synthesis of cellular c-di-GMP [e.g., nitric oxide (Plate and Marletta, 2012) and raffinose (Kim et al., 2016)], and iron chelating molecules [e.g., lactoferrin (Costerton et al., 1999)].

Lauroyl arginate ethyl (LAE) is a broad-spectrum, synthetic, non-oxidizing biocide (Kim and Park, 2016). It has a cationic arginine moiety and a hydrophobic lauric acid tail (Figure 1A), which facilitates the killing of microorganisms via the lysis of the cell membrane (Rodriguez et al., 2004). In the human body, LAE can be readily hydrolyzed into nutritional components such as lauroyl arginine and arginine (Hawkins et al., 2009). Thus, LAE has been widely employed as a preservative in the cosmetics, food, and beverage industries (Kuhnert, 2002; Becerril et al., 2013). Interestingly, LAE has been shown to inhibit biofilm formation by $P$. aeruginosa when used at a sub-growth inhibitory concentration (see the "Results" section). Nevertheless, the mechanism by which LAE prevents the development of a $P$. aeruginosa biofilm under various conditions is not yet known.

The primary goal of this study was to determine the mechanism of the anti-biofilm activity of LAE. Initially, we investigated global gene expression in $P$. aeruginosa treated with and without LAE at a sub-growth inhibitory concentration. Genes associated with iron acquisition and signaling were highly activated by LAE, which suggested the hypothesis that LAE generated an iron-limiting condition. To test this hypothesis, we measured the iron chelating activity of LAE and investigated the phenotype of $P$. aeruginosa cells under iron limitation (i.e., the twitching motility). The results suggested a possible anti-biofilm mechanism for LAE.

\section{MATERIALS AND METHODS}

\section{Bacterial Strain and Culture Condition}

Pseudomonas aeruginosa PA14 was used to test the effect of LAE (CDI, Hwaseong, South Korea) at a sub-growth inhibitory concentration. $P$. aeruginosa was cultured in a shaking incubator at $250 \mathrm{rpm}$ and $37^{\circ} \mathrm{C}$ in $\mathrm{AB}$ medium $(300 \mathrm{mM} \mathrm{NaCl}, 50 \mathrm{mM}$ $\mathrm{MgSO}_{4}, 0.2 \%$ vitamin-free casamino acids, $10 \mathrm{mM}$ potassium phosphate, $1 \mathrm{mM}$ L-arginine, and 1\% glucose, pH 7.5) (Kim and Park, 2013). The effect of LAE on growth was evaluated by inoculating an overnight culture of $P$. aeruginosa to an optical density (OD) at $595 \mathrm{~nm}$ of approximately 1.2 in $\mathrm{AB}$ medium supplemented with different concentrations of LAE. Growth was measured by the OD at $595 \mathrm{~nm}$ using a UVmini-1240 spectrophotometer (Shimadzu, Kyoto, Japan).

\section{Static Biofilm Formation Assay}

A static biofilm formation assay was used to test the effect of LAE on biofilm formation in $P$. aeruginosa. An overnight culture of $P$. aeruginosa was diluted in fresh $\mathrm{AB}$ medium (1:20) with and without $\operatorname{LAE}(10,50$, and $100 \mu \mathrm{M})$. Aliquots of $3 \mathrm{~mL}$ of the diluted culture were placed into borosilicate bottles and incubated at $37^{\circ} \mathrm{C}$ for $24 \mathrm{~h}$ without agitation. After $24 \mathrm{~h}$ incubation, the absorbance of the suspended cells in the borosilicate bottles was measured by the OD at $595 \mathrm{~nm}$ using a UVmini-1240 spectrophotometer (Shimadzu). After decanting the suspended cells, the biofilm cells that formed on the surface of the borosilicate bottles were stained for $30 \mathrm{~min}$ using crystal violet $(1.0 \%)$. The stained cells were then washed with deionized (DI) water to remove the residual crystal violet. The crystal violet in the biofilm cells was then eluted in ethanol (100\%). The absorbance of the eluted crystal violet was measured by the OD at $545 \mathrm{~nm}$ using a UVmini-1240 spectrophotometer (Shimadzu). The OD at $545 \mathrm{~nm}$ was normalized to the OD 
A<smiles>CCCCCCCCCCCC(=O)NC(CCCNC(N)=O)C(=O)OCC</smiles>

C

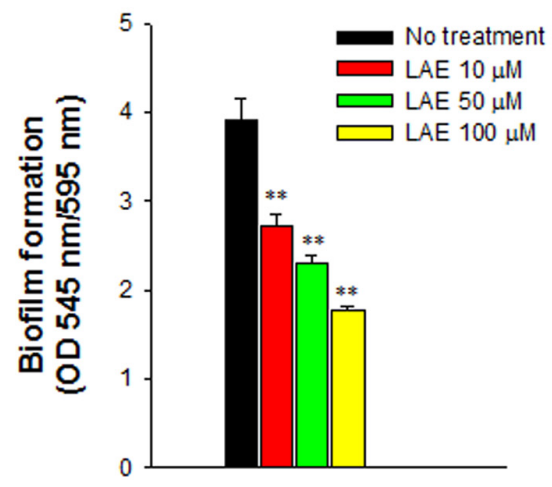

D
B
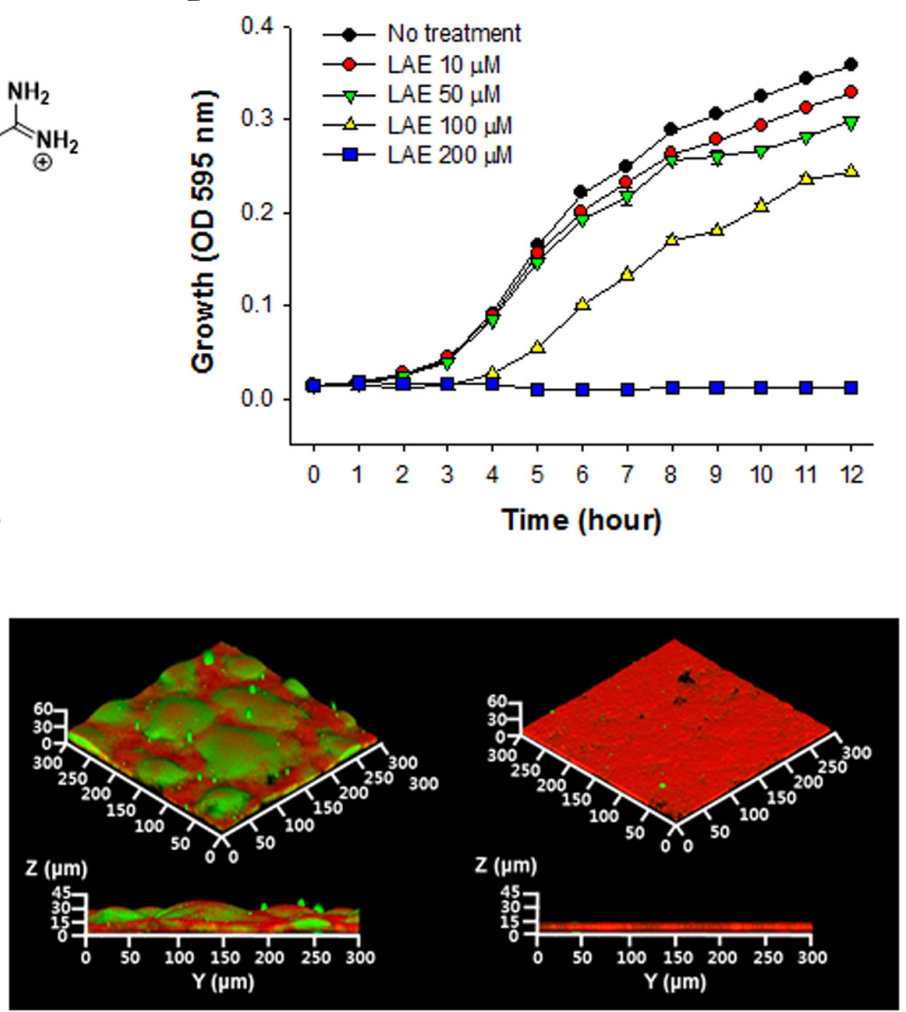

FIGURE 1 | Effect of lauroyl arginate ethyl (LAE) on Pseudomonas aeruginosa growth and biofilm formation. (A) Chemical structure of LAE. (B) Growth at different concentrations of LAE. Growth was analyzed by hourly measurements of the OD at $595 \mathrm{~nm}$. The error bars indicate the standard deviation of three measurements. (C) Biofilm formation in borosilicate bottles at different concentrations of LAE. Biofilm formation was analyzed by measuring the ratio of the OD at $545 \mathrm{~nm} / \mathrm{OD}$ at $595 \mathrm{~nm}$. The error bars indicate the standard deviation of three measurements, ${ }^{* *} P<0.005$ versus no treatment. (D) Confocal laser scanning microscopy (CLSM) images of 48-h biofilms formed on glass slides without (left images) and with $50 \mu \mathrm{M}$ LAE (right images). Green and red colors in the biofilms, respectively, indicate carbohydrates and proteins which were stained by Con-A and Ruby.

$595 \mathrm{~nm}$ to quantify the biofilm formation (O'Toole and Kolter, 1998b).

\section{Biofilm Formation in a Drip-Flow Reactor}

Biofilm formation in a drip-flow reactor (DFR-110, BioSurface Technologies, Bozeman, MT, United States) was evaluated using a procedure from a previous study (Goeres et al., 2009). A glass slide was immersed in a solution in which an overnight culture of $P$. aeruginosa $(1 \mathrm{~mL})$ was diluted in fresh $\mathrm{AB}$ medium $(19 \mathrm{~mL})$ in a petri dish. The slide was incubated at $37^{\circ} \mathrm{C}$ for $24 \mathrm{~h}$ to allow a biofilm to form on the slide. The slide was inserted into a dripflow biofilm reactor. Fresh $\mathrm{AB}$ medium with or without LAE $(50 \mu \mathrm{M})$ was continuously fed into the reactor by a peristaltic pump at $18 \mathrm{~mL} / \mathrm{h}$ at $37^{\circ} \mathrm{C}$ for $48 \mathrm{~h}$. After the reactor operation was complete, the slide was washed with phosphate-buffered saline (PBS) ( $\mathrm{pH}$ 7.2). The biofilm cells on the slide were stained with Con-A (Sigma-Aldrich, St. Louis, MO, United States) for $20 \mathrm{~min}$. After washing the slide with PBS again, the biofilm cells were stained with Ruby (Invitrogen, Carlsbad, CA, United States) for $20 \mathrm{~min}$. The stained biofilm cells were washed twice with DI water to remove the residual dye. Con-A and Ruby were, respectively, used to stain the carbohydrates and proteins in the biofilm. The biofilms were observed by confocal laser scanning microscopy (CLSM) (Carl Zeiss, LSM700, Jena, Germany). CLSM images were obtained using a 20x objective lens under a green fluorescence light for Con-A staining and red fluorescence light for Ruby staining. A stack of 3-D images was used to analyze the average thickness $(\mu \mathrm{m})$ and volume $\left(\mu \mathrm{m}^{3} / \mu \mathrm{m}^{2}\right)$ of a biofilm using the Comstat2 program from the ImageJ software (Heydorn et al., 2000; Vorregaard, 2008).

\section{Total RNA Extraction}

To carry out a microarray analysis, the total RNA was extracted using TRIzol reagent (Invitrogen, Carlsbad, CA, United States) from planktonic $P$. aeruginosa cells treated with and without LAE following the manufacturer's instructions. An overnight culture of $P$. aeruginosa was diluted in fresh $\mathrm{AB}$ medium to an $\mathrm{OD}$ at $595 \mathrm{~nm}=0.3$. Aliquots of $3 \mathrm{~mL}$ of the diluted culture were placed in borosilicate bottles with or without $50 \mu \mathrm{M}$ LAE and further cultured at $37^{\circ} \mathrm{C}$ for $4 \mathrm{~h}$ without agitation. After centrifugation at $8,000 \times g$ at $4^{\circ} \mathrm{C}$ for $10 \mathrm{~min}$, the culture supernatant was discarded. The $P$. aeruginosa pellets were resuspended in $1 \mathrm{~mL}$ of TRIzol reagent. The resuspended cells were mixed with $0.2 \mathrm{~mL}$ chloroform (99.9\%) and centrifuged at $12,000 \times g$ at $4^{\circ} \mathrm{C}$ for 
$15 \mathrm{~min}$. The aqueous phase was subsequently transferred to a $1.5 \mathrm{~mL}$ micro tube and $0.5 \mathrm{~mL}$ isopropyl alcohol (100\%) was added. The mixture was centrifuged at $12,000 \times g$ at $4{ }^{\circ} \mathrm{C}$ for $10 \mathrm{~min}$. The pellet was washed with $1 \mathrm{~mL}$ ethanol (75\%) and centrifuged again at $7,500 \times g$ at $4^{\circ} \mathrm{C}$ for $5 \mathrm{~min}$. The pellet (i.e., the total RNA) was dissolved in diethyl pyrocarbonatetreated water. The concentration and purity of the total RNA were, respectively, determined by the $\mathrm{OD}$ at $260 \mathrm{~nm}$ and the 260/280 nm ratio with an Agilent Bioanalyzer 2100 (Palo Alto, CA, United States). A total RNA concentration of greater than $100 \mathrm{ng} / \mu \mathrm{L}$ and an OD 260/280 nm ratio of 2.0 were used for subsequent analyses.

\section{Microarray Analysis}

Gene expression in $P$. aeruginosa cells treated with and without LAE was analyzed in duplicate by the SurePrint G3 custom gene expression array (Agilent Technology, Santa Clara, CA, United States). All of the procedures followed the manufacturer's protocols and were conducted at Macrogen Inc. (Seoul, South Korea). RNA labeling and hybridization were performed with the Agilent one-color microarray based gene expression analysis protocol (Ver. 6.5). The total RNA of each sample (200 ng) was amplified and labeled with Cy3-dCTP. The labeled cRNAs were purified using an RNeasy Mini kit (Qiagen, Valencia, CA, United States). The concentration of the labeled cRNAs (pmol Cy3/ $\mu$ g cRNA) was determined using a NanoDrop ND-1000 (Wilmington, DE, United States). The labeled cRNAs (600 ng) were fragmented by the addition of $5 \mu \mathrm{L}$ of a 10x blocking agent and $1 \mu \mathrm{L}$ of $25 \mathrm{x}$ fragmentation buffer, and were then incubated at $60^{\circ} \mathrm{C}$ for $30 \mathrm{~min}$. Finally, the labeled cRNAs were diluted with $25 \mu \mathrm{L}$ of $2 \mathrm{x}$ GE hybridization buffer. The hybridization solution $(50 \mu \mathrm{L})$ was dispensed into the gasket slide and assembled on a SurePrint G3 custom gene expression array $8 \times 60 \mathrm{~K}$ (Agilent Technology). The gasket slides were incubated for $17 \mathrm{~h}$ at $65^{\circ} \mathrm{C}$ in an Agilent hybridization oven (G2545A, Agilent Technology) and washed at room temperature using the Agilent protocol. The hybridized array was immediately scanned using an Agilent SureScan Microarray Scanner (Agilent Technologies). Array data export processing and analysis were performed using the Agilent Feature Extraction software v11.0.1.1.

\section{Gene Expression Omnibus (GEO) Accession Number}

The microarray data were deposited at the National Center for Biotechnology Information. The GEO accession number of the microarray data is GSE96844.

\section{Quantitative Reverse Transcription PCR (RT-qPCR)}

The expression of the genes related to the acquisition and storage of iron by $P$. aeruginosa treated with LAE was compared and quantified by RT-qPCR. The primer sets for the genes are listed in Supplementary Table S1. RT-qPCR was performed with a BioRad CFX-96 system (Bio-Rad, Hercules, CA, United States) with a one-step SYBR PrimeScript RT-PCR kit (Takara Bio, Otsu, Japan). The reaction mixture for RT-qPCR consisted of $10 \mu \mathrm{L}$ of 2x SYBR RT-PCR buffer, $1 \mu \mathrm{L}$ of PrimeScript enzyme mix, $0.4 \mu \mathrm{L}$ of 50x ROX Reference Dye I, 10 pmol of forward and reverse primers $(1.0 \mu \mathrm{L}$ of each solution), $100 \mathrm{ng} / \mu \mathrm{L}$ template RNA $(1 \mu \mathrm{L})$, and RNase free water to a final volume of $20 \mu \mathrm{L}$. The thermal conditions of the reaction for each of the target genes were: cDNA synthesis at $42^{\circ} \mathrm{C}$ for $5 \mathrm{~min}$, initial denaturation at $95^{\circ} \mathrm{C}$ for $10 \mathrm{~s}$ in step for reverse transcription, 40 denaturation cycles at $95^{\circ} \mathrm{C}$ for $5 \mathrm{~s}$ each, annealing at $60^{\circ} \mathrm{C}$ for $10 \mathrm{~s}$, and extension at $63^{\circ} \mathrm{C}$ for $34 \mathrm{~s}$. The fluorescence signal intensity was measured at the end of the extension step. A dissociation protocol was performed under conditions of $95^{\circ} \mathrm{C}$ for $15 \mathrm{~s}, 60^{\circ} \mathrm{C}$ for $1 \mathrm{~min}$, and $95^{\circ} \mathrm{C}$ for $15 \mathrm{~s}$ for the absence of a non-specific amplicon.

\section{Iron Chelation Assay}

Ferrous $\left(\mathrm{Fe}^{2+}\right)$ and ferric $\left(\mathrm{Fe}^{3+}\right)$ iron stock solutions were prepared with final concentrations from $20 \mu \mathrm{M}$ to $20 \mathrm{mM}$ by dissolving $\mathrm{FeCl}_{2}$ or $\mathrm{FeCl}_{3}$ in DI water. LAE-Fe chelation solutions were prepared by mixing $50 \mu \mathrm{L}$ of ferrous or ferric iron solution and $1 \mathrm{~mL}$ of LAE solution $(100 \mu \mathrm{M})$. The Fe/LAE molar ratios were $0,0.1,0.2,0.5,1.0,2.0,5.0$, and 10.0 . The mixed solution was gently shaken for $4 \mathrm{~h}$. Chromatographic analyses were performed using an Agilent 1260 series high-performance liquid chromatograph (HPLC) (Santa Clara, CA, United States) with a reverse-phase semi-preparative column (Phenomenex GeminiNX C18, $110 \AA, 150 \mathrm{~mm} \times 10 \mathrm{~mm}, 5 \mu \mathrm{m}$ ) for $40 \mathrm{~min}$ at a flow rate of $0.5 \mathrm{~mL} / \mathrm{min}$ with a $35 \%$ isocratic acetonitrile solution in DI water. The temperature of the autosampler and the column compartment were $45^{\circ} \mathrm{C}$. UV detection was carried out at a wavelength of $200 \mathrm{~nm}$. The HPLC system was injected with $10 \mu \mathrm{L}$ of each concentration of the mixed solution at least three times. The LAE peaks with a retention time of $17.7 \mathrm{~min}$ were used for the quantification analysis (Pezo et al., 2012).

\section{Twitching Motility Assay}

The twitching motility of the $P$. aeruginosa cells was assayed using the procedure of Singh et al. (2002). An overnight culture of $P$. aeruginosa was diluted in fresh $\mathrm{AB}$ medium to an $\mathrm{OD}$ at $595 \mathrm{~nm}$ of approximately 0.1 and supplemented with LAE. The diluted solution $(2 \mu \mathrm{L})$ was stab-inoculated on the bottom of a $1 \% \mathrm{AB}$ agar plate, which was incubated at room temperature for 2 days. The zone of twitching was measured with a Vernier caliper (Mitutoyo, Tsukuba, Japan).

\section{RESULTS}

\section{Inhibition of Biofilm Formation under Sub-growth Inhibitory Concentration at a LAE}

The growth of $P$. aeruginosa was evaluated at different concentrations of $\operatorname{LAE}(0,10,50,100$, and $200 \mu \mathrm{M})$ by measuring the $\mathrm{OD}$ at $595 \mathrm{~nm}$ for $12 \mathrm{~h}$ (Figure 1B). Growth decreased as the LAE concentration increased. $P$. aeruginosa showed relatively less growth inhibition at 10 and $50 \mu \mathrm{M}$ than at $100 \mu \mathrm{M}$, especially during the early period $(<5 \mathrm{~h})$. $P$. aeruginosa could not grow at $200 \mu \mathrm{M}$. P. aeruginosa biofilm 
formation was investigated in borosilicate bottles under static conditions under the sub-growth inhibitory conditions at LAE $(10,50$, and $100 \mu \mathrm{M})$. Figure $\mathbf{1 C}$ shows that biofilm formation (545 nm/595 nm) was reduced by $30.3-54.7 \%$ as the LAE concentration increased. Inhibition of biofilm formation was further verified by evaluating the biofilm development on a glass surface under flow conditions. Figure 1D shows CLSM images of 48 -h biofilms in the presence and absence of $50 \mu \mathrm{M}$ LAE. The biofilm in the absence of LAE had a characteristic thick, mushroom-like structure (average thickness $=33.8 \mu \mathrm{m}$, average volume $=17.5 \mu \mathrm{m}^{3} / \mu \mathrm{m}^{2}$ ), while the biofilm in the presence of LAE had a thin, flat structure (average thickness $=12.4 \mu \mathrm{m}$, average volume $=8.0 \mu \mathrm{m}^{3} / \mu \mathrm{m}^{2}$ ). Furthermore, the biofilm in the absence of LAE had more carbohydrates than the biofilm in the presence of LAE (i.e., there was an increase in the green color in the CLSM image of the biofilm in the absence of LAE). The decreased production of carbohydrates in the biofilm in the presence of LAE was verified by directly measuring the total carbohydrates in the biofilm matrix (Supplementary Figure S1).

\section{Analyses of Global Transcriptome at a Sub-growth Inhibitory Condition}

A global transcriptome analysis based on a microarray was conducted to identify the $P$. aeruginosa genes targeted by LAE at a sub-growth inhibitory concentration $(50 \mu \mathrm{M})$. After a quality check of the microarray data, 4,922 of 6,443 genes were selected for comparison of $P$. aeruginosa with and without LAE. Figure $\mathbf{2 A}$ is a heat map with hierarchical clustering that demonstrates that the relative gene expression between the two groups was significantly different, but did not differ within groups. A total of 1,061 of 4,922 genes were differentially expressed in the two groups with a twofold cut-off: 548 and 513 genes were, respectively, upor down-regulated (Supplementary Table S2). Classification of the differentially expressed genes according to metabolic functions (Stover et al., 2000) (Figure 2B) showed that diverse metabolic categories were affected by LAE, although the genes in hypothetical and unknown categories were the most abundant. If a fivefold change was used instead, 44 and 22 genes were, respectively, up-or down-regulated (Supplementary Table S3). Interestingly, many genes associated with cellular iron acquisition, signaling, and storage were found on the list. For example, the genes responsible for pyoverdine biosynthesis and transport ( $p v d$ and $f p v$ genes), and pyochelin biosynthesis and transport ( $p c h$ and $f p t$ genes) were up-regulated, while the activity of bacterioferritin biosynthesis genes ( $b f r$ ) was down-regulated. The fold-changes in expressions of the pyochelin-, pyoverdine-, and bacterioferritin-associated genes are summarized in Table $\mathbf{1}$.

To verify the expression of the iron acquisition, signaling, and storage genes, RT-qPCR was conducted for representative pyochelin, pyoverdine, and bacterioferritin genes ( $p$ ch $A, p c h B$, $p c h F, p c h R, f p t A, p v d D, p v d E, p v d L, p v d Q, p v d R, p v d S, f p v A$, and $b f r B$ ), as shown in Figure 3 . The results concur with
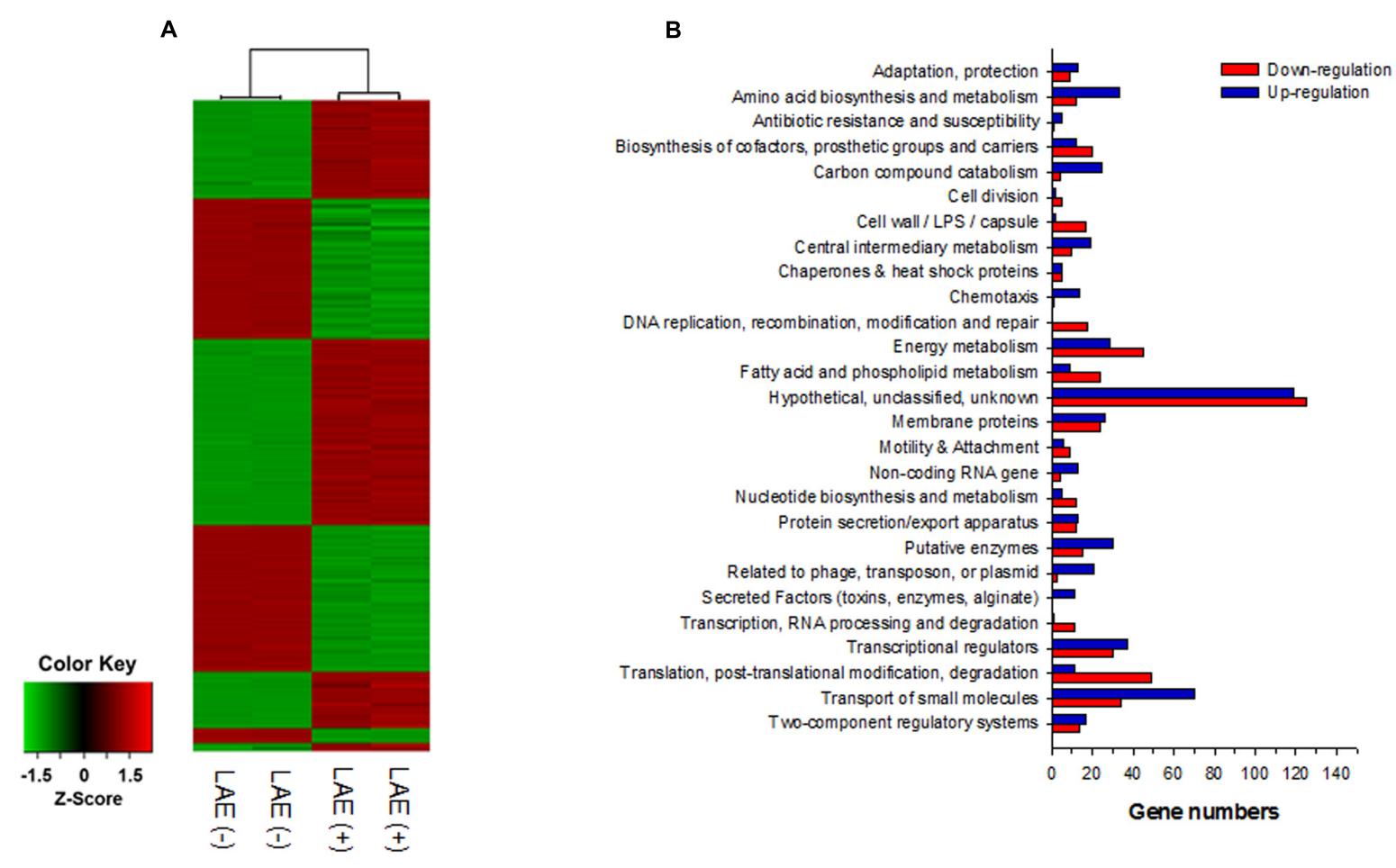

FIGURE 2 | Global transcriptome analysis for P. aeruginosa with and without 50 mM LEA. (A) Heatmap of the expression of 4,922 genes with hierarchical clustering. (B) Classification of up- and down-regulated genes based on metabolic function. 
TABLE 1 | Fold-changes in expression of the pyochelin, pyoverdine, and bacterioferritin genes in response to 50 mM lauroyl arginate ethyl (LAE).

\begin{tabular}{|c|c|c|c|}
\hline ID & Gene name & Gene product & Fold-change \\
\hline \multicolumn{4}{|c|}{ Pyochelin system } \\
\hline PA14_09340 & fptA & Fe(III)-pyochelin outer membrane receptor & 4.8 \\
\hline PA14_09350 & fptB & Hypothetical protein & 4.9 \\
\hline PA14_09380 & $f p t X$ & Probable transporter & 7.1 \\
\hline PA14_09210 & pchA & Salicylate biosynthesis isochorismate synthase & 6.4 \\
\hline PA14_09220 & pchB & Salicylate biosynthesis protein PchB & 7.8 \\
\hline PA14_09230 & pchC & Pyochelin biosynthetic protein PchC & 5.4 \\
\hline PA14_09240 & $\operatorname{pchD}$ & Pyochelin biosynthetic protein PchD & 6.7 \\
\hline PA14_09260 & pchR & Transcriptional regulator PchR & 4.7 \\
\hline PA14_09270 & pchE & Dihydroaeruginoic acid synthetase & 5.4 \\
\hline PA14_09280 & pchF & Pyochelin synthetase & 7.6 \\
\hline PA14_09290 & $p c h G$ & Pyochelin biosynthetic protein PchG & 5.5 \\
\hline PA14_09300 & $\mathrm{pchH}$ & Probable ATP-binding component of ABC transporter & 4.0 \\
\hline PA14_09320 & pchl & Probable ATP-binding component of ABC transporter & 4.4 \\
\hline \multicolumn{4}{|c|}{ Pyoverdine system } \\
\hline PA14_33680 & $f p v A$ & Ferripyoverdine receptor & 3.6 \\
\hline PA14_09970 & $f p v B$ & Second ferric pyoverdine receptor FpvB & 1.6 \\
\hline PA14_33810 & $p v d A$ & I-Ornithine N5-oxygenase & 5.3 \\
\hline PA14_33650 & $p v d D$ & Pyoverdine synthetase D & 2.2 \\
\hline PA14_33690 & $p v d E$ & Pyoverdine biosynthesis protein PvdE & 1.7 \\
\hline PA14_33700 & $p v d F$ & Pyoverdine synthetase F & 1.9 \\
\hline PA14_33270 & $p v d G$ & Protein PvdG & 2.6 \\
\hline PA14_33500 & $p v d H$ & I-2,4-diaminobutyrate:2-ketoglutarate 4-aminotransferase, PvdH & 5.6 \\
\hline PA14_33610 & pvdl & Probable non-ribosomal peptide synthetase & 4.4 \\
\hline PA14_33630 & pvdJ & Protein PvdJ & 2.2 \\
\hline PA14_33280 & $p v d L$ & Peptide synthase PvdL & 3.3 \\
\hline PA14_33720 & $p v d N$ & Protein PvdN & 2.5 \\
\hline PA14_33710 & $p v d O$ & Protein PvdO & 6.5 \\
\hline PA14_33740 & $p v d P$ & Protein PvdP & 2.7 \\
\hline PA14_33820 & $p v d Q$ & 3-Oxo-C12-homoserine lactone acylase PvdQ & 4.1 \\
\hline PA14_33260 & pvdS & Sigma factor PvdS & 2.9 \\
\hline PA14_39800 & & ECF sigma factor, Feml & 3.3 \\
\hline \multicolumn{4}{|c|}{ Other iron acquisition genes } \\
\hline PA14_09160 & bfrA & Bacterioferritin & -15.1 \\
\hline PA14_18670 & $b f r B$ & Bacterioferritin & -33.5 \\
\hline PA14_72970 & ton $B$ & TonB protein & 8.9 \\
\hline
\end{tabular}

the microarray results: the pyochelin and pyoverdine-associated genes were up-regulated (1.5- to 5.1-folds), except for $p v d L$ (0.98fold), and a bacterioferritin gene $(b f r B)$ was down-regulated (0.3-fold). The expression of the proC housekeeping gene was not affected by LAE $(P>0.05)$. A noteworthy result was the higher activation of the pch genes than the pvd genes $(P<0.05)$.

\section{Iron Chelation Activity by LAE}

The iron chelation activity of LAE was measured by HPLC, by determining the unbound LAE as the ferrous or ferric iron concentration was increased (the ratio of the iron to the LAE concentration was varied between 0 and 10). Figure 4 shows that the LAE signals in the HPLC chromatograms decreased asymptotically with an increase in the iron concentration when the LAE concentration was fixed at $100 \mu \mathrm{M}$, demonstrating that LAE could chelate iron. However, LAE appeared to be a weak iron chelator because he HPLC analysis indicated that the LAE signal did not show a further decrease, even when the ratio was as high as 10 (i.e., there was a 53.7 and $55.5 \%$ reduction in ferrous and ferric iron, respectively, at a ratio of 10). Another characteristic of LAE is its specificity. LAE had chelating activity for both ferric iron and ferrous iron. We also noted that the putative genes for ferrous iron uptake ( $f e o$ ) (Cartron et al., 2006) were up-regulated in response to LAE treatment in the microarray analysis (Supplementary Tables S2, S3).

\section{Effect of LAE on Twitching Motility}

Twitching motility is a translocation mode of P. aeruginosa, and it involves type IV pili which can attach to a surface and pull $P$. aeruginosa in a forward direction (Burrows, 2012). Figure 5 


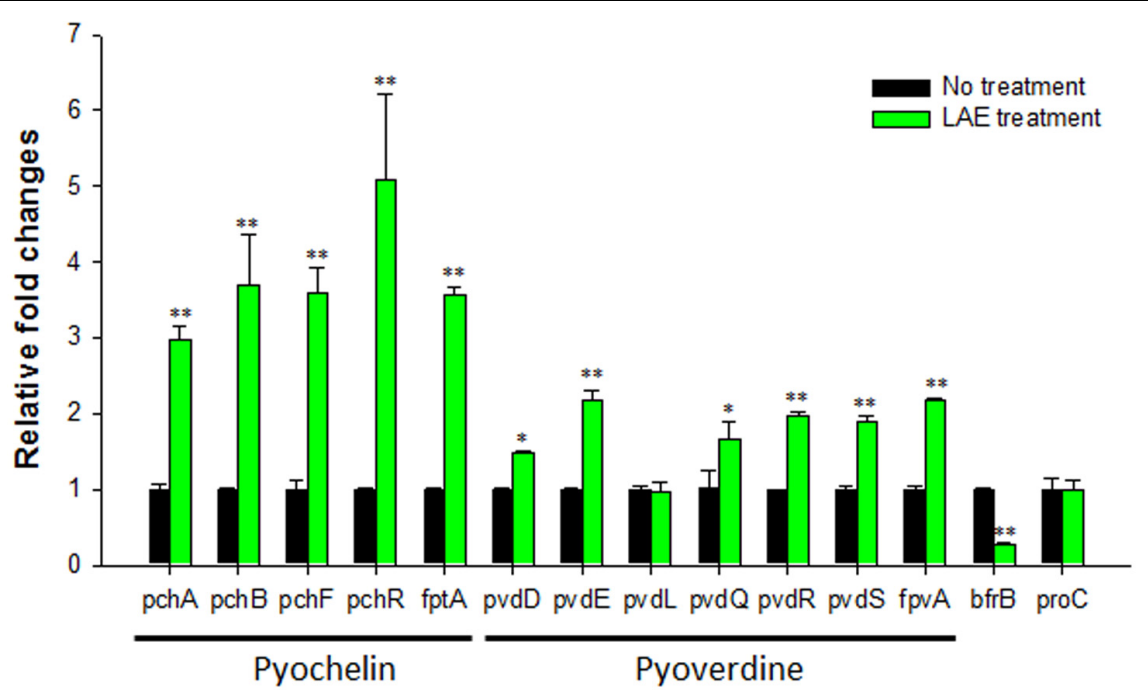

FIGURE 3 | Effect of LAE on the expression of iron acquisition, signaling, and storage genes in $P$. aeruginosa with and without 50 mM LEA. The fold-change was defined as the relative copy number of the cDNA of each gene normalized by the copy number of the cDNA of the corresponding gene in the absence of LAE. The error bars indicate the standard deviation of three measurements. ${ }^{*} P<0.05$ versus no treatment, ${ }^{* *} P<0.005$ versus no treatment.

A

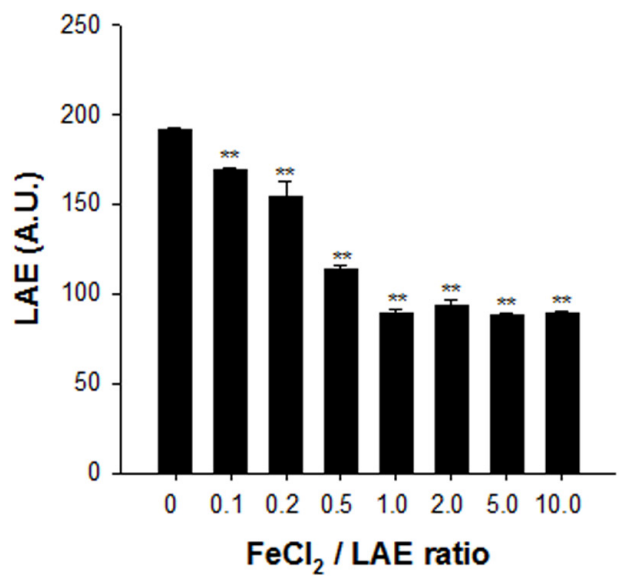

B

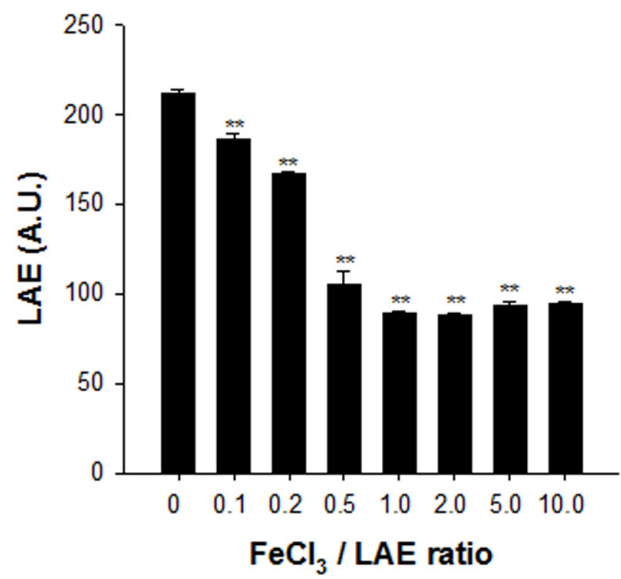

FIGURE 4 | Analysis of iron chelation by LAE at different ratios of iron and LAE. The LAE concentration was fixed at $100 \mathrm{mM}$. (A) Ferrous iron (Fe $2+$ ) chelation by $L A E$. (B) Ferric iron $\left(\mathrm{Fe}^{3+}\right)$ chelation by $L A E$. The error bars indicate the standard deviation of three measurements. ${ }^{* *} P<0.005$ versus no treatment of ferric or ferrous iron.

shows that LAE stimulated the twitching motility by $146-203 \%$ at the agar and plastic interfaces in a concentration-dependent manner $(10-100 \mu \mathrm{M})$. On the other hand, the twitching motility was gradually decreased by an increase in the supplemental ferrous or ferric iron concentration.

\section{DISCUSSION}

Iron is essential for bacterial growth, primarily because it acts as a cofactor for enzymes related to oxygen metabolism, electron transport, and nucleic acid synthesis (Braud et al., 2009). However, iron is toxic to bacteria at higher concentrations due to the formation of reactive oxygen species (Cornelis and Andrews, 2012). Therefore, it is important for the bacteria to maintain an optimal intracellular iron concentration. Similar to the situation in many other bacteria, iron homeostasis in P. aeruginosa is regulated by the Fur protein (Cornelis et al., 2009). Extracellular iron cannot be transported into the cytoplasm via passive transport (Banin et al., 2005). Therefore, many bacteria use endogenous siderophores to transport extracellular iron. Pyochelin and pyoverdine are representative ferric iron chelating molecules (i.e., siderophores) for intracellular iron acquisition, and are secreted by certain pseudomonads including P. aeruginosa (Banin et al., 2005). Pyoverdine is reported to have a higher affinity for ferric iron than pyochelin (Banin 


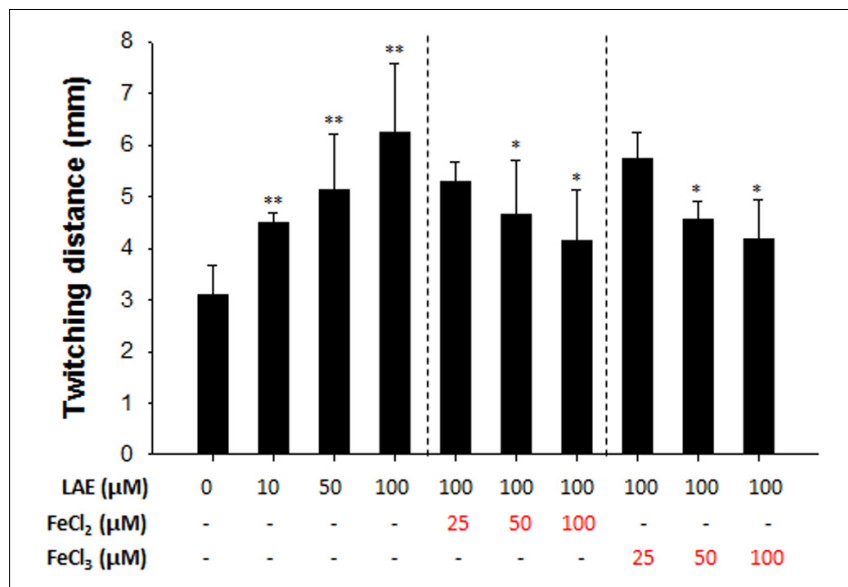

FIGURE 5 | Effect of LAE on P. aeruginosa twitching motility. Twitching distances were estimated by measuring the radius of the cell clusters on plates. The error bars indicate the standard deviation of three measurements ${ }^{*} P<0.05$ versus $100 \mathrm{mM}$ LAE treatment, ${ }^{* *} P<0.005$ versus no treatment.

et al., 2005). Figure 6 shows that, for ferric iron transport into the cytoplasm, the ferric iron initially binds to extracellularly secreted pyoverdine or pyochelin, and the bound ferric iron is transported across the outer membrane via an energycoupled TonB-dependent receptor (i.e., FpvA for pyoverdine and FptA for pyochelin). After binding to a periplasmic binding protein in the periplasmic space, the bound ferric iron is transported across the inner membrane via an ATP-binding cassette transporter $(\mathrm{ABC})$ transporter, while the ferric ion separates from the pyoverdine or pyochelin and is reduced to ferrous iron. In the cytoplasm, ferrous iron is used for synthesis of enzymes or for storage. Excess ferrous iron is bound to a Fur protein and regulates the genes involved in iron acquisition (e.g., the pyoverdine and pyochelin systems), biofilm formation, synthesis of small RNAs, etc., directly or via extracytoplasmic sigma factors (ECF $\sigma$ factors) or other regulators (Cornelis et al., 2009). For example, the Fur protein, when bound to ferrous iron, represses the genes for the ECF $\sigma$ factors involved in the pyoverdine system (e.g., pvdS and $f p v I)$ and represses genes for the transcriptional regulators of the pyochelin system (e.g., $p c h R$ ). The genes for pyoverdine synthesis and uptake are additionally regulated by the ECF $\sigma$ factors (PvdS and FpvI) that are bound to an anti-sigma factor (FpvR) (Edgar et al., 2014). Upon the binding of ferric iron to FpvA, a signal is transmitted to FpvR, which enables the FpvR to release PvdS or FpvI, and the released PvdS or FvpI, respectively, activates the $p v d$ genes or the $f p v A$ gene.

In this study, the microarray and RT-qPCR results demonstrated that the genes associated with iron acquisition, signaling, and storage are targeted by LAE at the sub-growth inhibitory concentration. Furthermore, an experiment that measured free LAE molecules by increasing the ratio of iron to LAE demonstrated that LAE chelates iron, which led to the hypothesis that LAE generated iron-limiting conditions. To test this hypothesis, we evaluated the twitching motility of
P. aeruginosa. Singh et al. (2002) reported that iron-limiting conditions generated by the presence of the iron chelating molecule deferoxamine showed increased twitching motility in $P$. aeruginosa. Therefore, we conducted an experiment to test whether exposure to LAE increases the twitching motility, which showed that LAE stimulated twitching motility in proportion to the supplemental ferrous or ferric iron present. Furthermore, the twitching motility gradually decreased as the supplemental iron concentration increased. These results suggest that LAE created iron-limiting conditions, and the iron-limiting conditions were relieved by the supplemental iron.

It is reasonable to speculate that the iron-limiting conditions led to the activation of the genes associated with the pyoverdine and pyochelin systems, allowing better uptake of extracellular iron. Iron-limiting conditions generated by the LAE treatment would hinder the transport of ferric iron into the cytoplasm in $P$. aeruginosa, which in turn would cause the ferrous iron to become unbound from the Fur protein. The native Fur protein would relieve the repression of the genes for the pyoverdine and pyochelin systems (e.g., $p v d S$, $f p v I$, and $p c h R$ ). Interestingly, in our study, the pyochelin-associated genes were more highly activated than the pyoverdine-associated genes (Figure 3). Two previous studies on global gene expression in $P$. aeruginosa in response to iron starvation (Ochsner et al., 2002; Palma et al., 2003) showed the same trend. These results were contrary to our expectations, because a high-affinity siderophore (i.e., pyoverdine) would be expected to show higher expression in order to bind the limited extracellular iron, as opposed to an increase in the expression of a low-affinity siderophore (i.e., pyochelin). The higher expression of the pyochelin-associated genes suggests that pyochelin may have other functions besides iron uptake. Several studies support this speculation. Pyochelin has been reported to remove iron bound to transferrin, a serum glycoprotein, to secure iron (Sriyosachati and Cox, 1986). This may contribute to the growth and virulence of $P$. aeruginosa (Cox, 1982). In addition, pyochelin catalyzes the formation of free radicals that can damage host tissues (Britigan et al., 1994).

In $P$. aeruginosa, iron acts as a signal for biofilm development as well as a micronutrient for growth. If $P$. aeruginosa cells are under iron-limiting conditions or cannot acquire iron, they cannot form a mature biofilm. Singh et al. (2002) demonstrated that biofilm formation in $P$. aeruginosa was prevented by lactoferrin, a component of human mucosal secretion that chelates iron. We also found that LAE chelates ferric and ferrous iron, and that $P$. aeruginosa treated with LAE forms thin biofilms, similar to the results of a study by Singh et al. (2002). On the other hand, Banin et al. (2005) found that mutants that could not produce pyoverdine or pyochelin could not form a mature biofilm. In addition, they demonstrated that a Fur mutant could form a mushroom-like biofilm even under iron-limiting conditions. From their results, they claimed that intracellular iron is essential for the development of a mature biofilm, and that iron is a signal for biofilm development that acts via the Fur regulator. In this regard, our results suggest that LAE can be an effective inhibitor of iron signaling for biofilm development, possibly via the Fur regulator. 


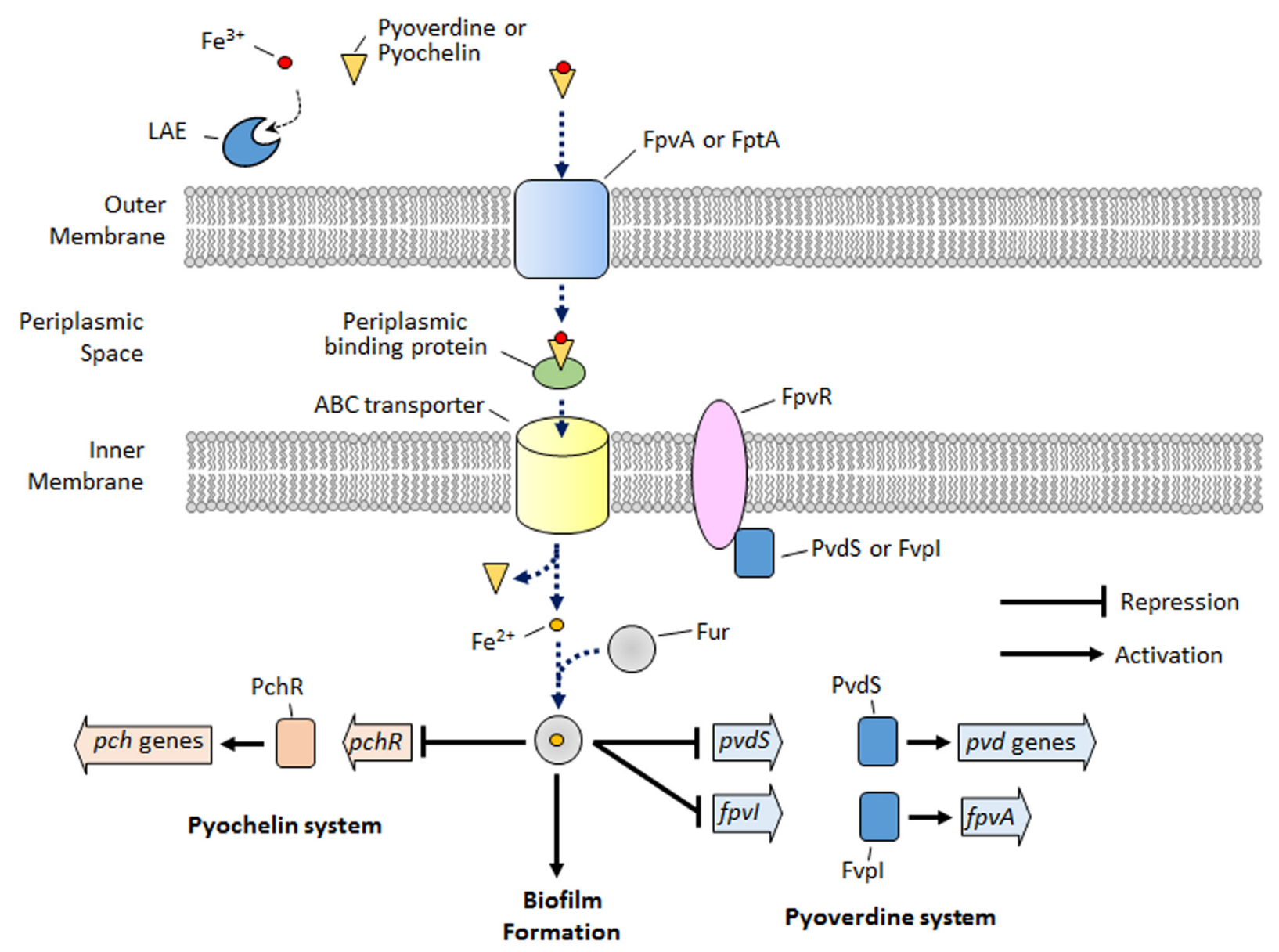

FIGURE 6 | Schematic depicting the simplified iron signaling for the pyoverdine/pyochelin system and biofilm formation in $P$. aeruginosa. LAE competes with pyoverdine or pyochelin to chelate extracellular iron. Upon iron starvation via iron chelation by LAE, $P$. aeruginosa activates the genes for the pyoverdine and pyochelin systems and inhibits biofilm formation, which is the opposite condition to that depicted in the schematic.

Lauroyl arginate ethyl was expected to possess iron chelating activity because it contains donor groups found in common ferric (e.g., deferoxamine and deferiprone) and ferrous (e.g., ferrozine and phenanthroline) iron chelators (Haas and Franz, 2009). Figure 1A shows that LAE contains two types of donor groups that can potentially form ferric and ferrous iron chelate complexes: the two carbonyl groups (ester and amide) with a 1,4-relationship and a guanidine group. On the other hand, since ferric iron is a harder acid than ferrous iron, the harder ligands (e.g., hydroxamate, phenolate, and catecholate) with electronegative oxygen donor groups would preferentially coordinate with ferric iron rather than with ferrous iron (Martell et al., 1996). However, the softer ligands (e.g., ethylenediaminetetraacetic acid, trimethylenedinitrilotetraacetic acid) with both oxygen and nitrogen donor groups increase the stability of ferrous chelate, although they still have a slight preference for ferric iron. Because LAE possesses both oxygen donor groups (i.e., ester and amide groups) and a nitrogen donor group (i.e., guanidine), it might act as a softer ligand, thus increasing the relative stability of the ferrous-LAE complex.
The inhibition of biofilm formation in $P$. aeruginosa by LAE has important implications in medicine and industry. Colonization by $P$. aeruginosa in the form of biofilms on various surfaces (e.g., lung, catheters, water filters, etc.) frequently causes serious problems. However, it is difficult to eliminate $P$. aeruginosa biofilms due to their resistance to antibiotics and biocides. Our finding suggests that LAE can mitigate such problems by retarding biofilm formation. In line with reports on the vulnerability of biofilm cells to antibiotics in the presence of a biofilm inhibitor (Brackman et al., 2011), LAE might also be used to kill biofilm cells by administering the LAE along with antibiotics. In addition, the low toxicity of LAE (Ruckman et al., 2004) is an advantage in medical applications.

\section{CONCLUSION}

In this study, we found that LAE, a synthetic biocide, inhibited biofilm development in P. aeruginosa at sub-growth inhibitory concentrations. LAE activated the genes involved in iron acquisition (e.g., the pyoverdine and pyochelin related genes) and 
increased twitching motility, due to the low availability of iron to $P$. aeruginosa because LAE chelated the iron. We can infer that low levels of cellular iron blocked the iron signal that led to biofilm formation. We expect that LAE can aid in the treatment of $P$. aeruginosa biofilms in medical and industrial settings as an effective biofilm inhibitor.

\section{AUTHOR CONTRIBUTIONS}

H-DP and YB conceived and designed the experiments; T-SK, $\mathrm{S}-\mathrm{YH}$, and BP performed the experiments; H-DP, YB, and T-SK analyzed the data; H-DP, T-SK, and YB wrote the paper; all authors approved the final version of the manuscript.

\section{REFERENCES}

Banin, E., Vasil, M. L., and Greenberg, E. P. (2005). Iron and Pseudomonas aeruginosa biofilm formation. Proc. Natl. Acad. Sci. U.S.A. 102, 11076-11081. doi: 10.1073/pnas.0504266102

Becerril, R., Manso, S., Nerin, C., and Gómez-Lus, R. (2013). Antimicrobial activity of Lauroyl arginate ethyl (LAE), against selected food-borne bacteria. Food Control 32, 404-408. doi: 10.1016/j.foodcont.2013.01.003

Borlee, B. R., Goldman, A. D., Murakami, K., Samudrala, R., Wozniak, D. J., and Parsek, M. R. (2010). Pseudomonas aeruginosa uses a cyclic-di-GMPregulated adhesin to reinforce the biofilm extracellular matrix. Mol. Microbiol. 75, 827-842. doi: 10.1111/j.1365-2958.2009.06991.x

Brackman, G., Cos, P., Maes, L., Nelis, H. J., and Coenye, T. (2011). Quorum sensing inhibitors increase the susceptibility of bacterial biofilms to antibiotics in vitro and in vivo. Antimicrob. Agents Chemother. 55, 2655-2661. doi: 10.1128/AAC.00045-11

Braud, A., Hannauer, M., Mislin, G. L., and Schalk, I. J. (2009). The Pseudomonas aeruginosa pyochelin-iron uptake pathway and its metal specificity. J. Bacteriol. 191, 3517-3525. doi: 10.1128/JB.00010-09

Britigan, B. E., Rasmussen, G. T., and Cox, C. D. (1994). Pseudomonas siderophore pyochelin enhances neutrophil-mediated endothelial cell injury. Am. J. Physiol. Lung Cell Mol. Physiol. 266, L192-L198.

Burrows, L. L. (2012). Pseudomonas aeruginosa twitching motility: type IV pili in action. Annu. Rev. Microbiol. 66, 493-520. doi: 10.1146/annurev-micro092611-150055

Cartron, M. L., Maddocks, S., Gillingham, P., Craven, C. J., and Andrews, S. C. (2006). Feo - transport of ferrous iron into bacteria. Biometals 19, 143-157. doi: 10.1007/s10534-006-0003-2

Cornelis, P., and Andrews, S. C. (2012). "Regulation of iron homeostasis in bacteria," in Bacterial Regulatory Networks, ed. A. A. M. Filloux (Norfolk, VA: Caister Academic Press).

Cornelis, P., Matthijs, S., and Van Oeffelen, L. (2009). Iron uptake regulation in Pseudomonas aeruginosa. Biometals 22, 15-22. doi: 10.1007/s10534-008-9193-0

Costerton, J. W., Stewart, P. S., and Greenberg, E. P. (1999). Bacterial biofilms: a common cause of persistent infections. Science 284, 1318-1322. doi: 10.1126/ science.284.5418.1318

Cox, C. D. (1982). Effect of pyochelin on the virulence of Pseudomonas aeruginosa. Infect. Immun. 36, 17-23.

Davies, D. G., Parsek, M. R., Pearson, J. P., Iglewski, B. H., Costerton, J. T., and Greenberg, E. P. (1998). The involvement of cell-to-cell signals in the development of a bacterial biofilm. Science 280, 295-298. doi: 10.1126/science. 280.5361.295

Dong, Y.-H., and Zhang, L.-H. (2005). Quorum sensing and quorum-quenching enzymes. J. Microbiol. 43, 101-109.

Driscoll, J. A., Brody, S. L., and Kollef, M. H. (2007). The epidemiology, pathogenesis and treatment of Pseudomonas aeruginosa infections. Drugs 67, 351-368. doi: 10.2165/00003495-200767030-00003

Edgar, R. J., Xu, X., Shirley, M., Konings, A. F., Martin, L. W., Ackerley, D. F., et al. (2014). Interactions between an anti-sigma protein and two sigma factors that

\section{FUNDING}

This research was funded by grants from the National Research Foundation (NRF) of Korea (2015R1D1A1A09057657 and 2014R1A4A1007304).

\section{SUPPLEMENTARY MATERIAL}

The Supplementary Material for this article can be found online at: http://journal.frontiersin.org/article/10.3389/fmicb. 2017.00970/full\#supplementary-material

FIGURE S1 | Total proteins and total carbohydrates in biofilms with and without LAE. ${ }^{*} P<0.05$ versus no treatment.

regulate the pyoverdine signaling pathway in Pseudomonas aeruginosa. BMC Microbiol. 14:287. doi: 10.1186/s12866-014-0287-2

Goeres, D. M., Hamilton, M. A., Beck, N. A., Buckingham-Meyer, K., Hilyard, J. D., Loetterle, L. R., et al. (2009). A method for growing a biofilm under low shear at the air-liquid interface using the drip flow biofilm reactor. Nat. Protoc. 4, 783-788. doi: 10.1038/nprot.2009.59

Haas, K. L., and Franz, K. J. (2009). Application of metal coordination chemistry to explore and manipulate cell biology. Chem. Rev. 109, 4921-4960. doi: 10.1021/ cr900134a

Hawkins, D., Rocabayera, X., Ruckman, S., Segret, R., and Shaw, D. (2009). Metabolism and pharmacokinetics of ethyl $\mathrm{N}^{\alpha}$-lauroyl-L-arginate hydrochloride in human volunteers. Food Chem. Toxicol. 47, 2711-2715. doi: 10.1016/j.fct.2009.07.028

He, J., Baldini, R. L., Deziel, E., Saucier, M., Zhang, Q., Liberati, N. T., et al. (2004). The broad host range pathogen Pseudomonas aeruginosa strain PA14 carries two pathogenicity islands harboring plant and animal virulence genes. Proc. Natl. Acad. Sci. U.S.A. 101, 2530-2535. doi: 10.1073/pnas.0304622101

Hentzer, M., Riedel, K., Rasmussen, T. B., Heydorn, A., Andersen, J. B., Parsek, M. R., et al. (2002). Inhibition of quorum sensing in Pseudomonas aeruginosa biofilm bacteria by a halogenated furanone compound. Microbiology 148, 87-102. doi: 10.1099/00221287-148-1-87

Hentzer, M., Wu, H., Andersen, J. B., Riedel, K., Rasmussen, T. B., Bagge, N., et al. (2003). Attenuation of Pseudomonas aeruginosa virulence by quorum sensing inhibitors. EMBO J. 22, 3803-3815. doi: 10.1093/emboj/cdg366

Heydorn, A., Nielsen, A. T., Hentzer, M., Sternberg, C., Givskov, M., Ersbøll, B. K., et al. (2000). Quantification of biofilm structures by the novel computer program COMSTAT. Microbiology 146, 2395-2407. doi: 10.1099/00221287146-10-2395

Jimenez, P. N., Koch, G., Thompson, J. A., Xavier, K. B., Cool, R. H., and Quax, W. J. (2012). The multiple signaling systems regulating virulence in Pseudomonas aeruginosa. Microbiol. Mol. Biol. Rev. 76, 46-65. doi: 10.1128/MMBR.05007-11

Kim, H.-S., Cha, E., Kim, Y., Jeon, Y. H., Olson, B. H., Byun, Y., et al. (2016). Raffinose, a plant galactoside, inhibits Pseudomonas aeruginosa biofilm formation via binding to LecA and decreasing cellular cyclic diguanylate levels. Sci. Rep. 6:25318. doi: 10.1038/srep25318

Kim, H.-S., Lee, S.-H., Byun, Y., and Park, H.-D. (2015). 6-Gingerol reduces Pseudomonas aeruginosa biofilm formation and virulence via quorum sensing inhibition. Sci. Rep. 5:8656. doi: 10.1038/srep08656

Kim, H.-S., and Park, H.-D. (2013). Ginger extract inhibits biofilm formation by Pseudomonas aeruginosa PA14. PLoS ONE 8:e76106. doi: 10.1371/journal.pone. 0076106

Kim, T.-S., and Park, H.-D. (2016). Lauroyl arginate ethyl: an effective antibiofouling agent applicable for reverse osmosis processes producing potable water. J. Membrane Sci. 507, 24-33. doi: 10.1016/j.memsci.2016.01.056

Kuhnert, P. (2002). "Foods, 3. Food additives," in Ullmann's Encyclopedia of Industrial Chemistry, ed. B. Elvers (Hoboken, NJ: John Wiley and Sons, Inc.). doi: 10.1002/14356007.a11_561.pub2

Martell, A. E., Motekaitis, R. J., Chen, D., Hancock, R. D., and McManus, D. (1996). Selection of new Fe (III)/Fe (II) chelating agents as catalysts for the 
oxidation of hydrogen sulfide to sulfur by air. Can. J. Chem. 74, 1872-1879. doi: $10.1139 / \mathrm{v} 96-210$

Ochsner, U. A., Wilderman, P. J., Vasil, A. I., and Vasil, M. L. (2002). GeneChip ${ }^{\circledR}$ expression analysis of the iron starvation response in Pseudomonas aeruginosa: identification of novel pyoverdine biosynthesis genes. Mol. Microbiol. 45, 1277-1287. doi: 10.1046/j.1365-2958.2002.03084.x

O’Toole, G. A., and Kolter, R. (1998a). Flagellar and twitching motility are necessary for Pseudomonas aeruginosa biofilm development. Mol. Microbiol. 30, 295-304. doi: 10.1046/j.1365-2958.1998.01062.x

O’Toole, G. A., and Kolter, R. (1998b). Initiation of biofilm formation in Pseudomonas fluorescens WCS365 proceeds via multiple, convergent signalling pathways: a genetic analysis. Mol. Microbiol. 28, 449-461. doi: 10.1046/j.13652958.1998.00797.x

Palma, M., Worgall, S., and Quadri, L. E. (2003). Transcriptome analysis of the Pseudomonas aeruginosa response to iron. Arch. Microbiol. 180, 374-379. doi: 10.1007/s00203-003-0602-z

Percival, S. L., Suleman, L., Vuotto, C., and Donelli, G. (2015). Healthcareassociated infections, medical devices and biofilms: risk, tolerance and control. J. Med. Microbiol. 64, 323-334. doi: 10.1099/jmm.0.000032

Pezo, D., Navascués, B., Salafranca, J., and Nerín, C. (2012). Analytical procedure for the determination of Ethyl Lauroyl Arginate (LAE) to assess the kinetics and specific migration from a new antimicrobial active food packaging. Anal. Chim. Acta 745, 92-98. doi: 10.1016/S0168-8278(00)80240-8

Plate, L., and Marletta, M. A. (2012). Nitric oxide modulates bacterial biofilm formation through a multicomponent cyclic-di-GMP signaling network. Mol. Cell 46, 449-460. doi: 10.1016/j.molcel.2012.03.023

Rodriguez, E., Seguer, J., Rocabayera, X., and Manresa, A. (2004). Cellular effects of monohydrochloride of L-arginine, $\mathrm{N}^{\alpha}$-lauroyl ethylester (LAE) on exposure to Salmonella typhimurium and Staphylococcus aureus. J. Appl. Microbiol. 96, 903-912. doi: 10.1111/j.1365-2672.2004.02207.x
Ruckman, S. A., Rocabayera, X., Borzelleca, J. F., and Sandusky, C. B. (2004). Toxicological and metabolic investigations of the safety of $\mathrm{N}-\alpha$-LauroylL-arginine ethyl ester monohydrochloride (LAE). Food Chem. Toxicol. 42, 245-259. doi: 10.1016/j.fct.2003.08.022

Singh, P. K., Parsek, M. R., Greenberg, E. P., and Welsh, M. J. (2002). A component of innate immunity prevents bacterial biofilm development. Nature 417, 552-555. doi: 10.1038/417552a

Sriyosachati, S., and Cox, C. D. (1986). Siderophore-mediated iron acquisition from transferrin by Pseudomonas aeruginosa. Infect. Immun. 52, $885-891$.

Stover, C., Pham, X., Erwin, A., Mizoguchi, S., Warrener, P., Hickey, M., et al. (2000). Complete genome sequence of Pseudomonas aeruginosa PAO1, an opportunistic pathogen. Nature 406, 959-964. doi: 10.1038/35 023079

Vorregaard, M. (2008). COMSTAT2-A Modern 3D Image Analysis Environment for Biofilms. Master's thesis, Technical University of Denmark, Kongens Lyngby.

Weinstein, R. A., and Darouiche, R. O. (2001). Device-associated infections: a macroproblem that starts with microadherence. Clin. Infect. Dis. 33, 1567-1572. doi: $10.1086 / 323130$

Conflict of Interest Statement: The authors declare that the research was conducted in the absence of any commercial or financial relationships that could be construed as a potential conflict of interest.

Copyright (C) 2017 Kim, Ham, Park, Byun and Park. This is an open-access article distributed under the terms of the Creative Commons Attribution License (CC BY). The use, distribution or reproduction in other forums is permitted, provided the original author(s) or licensor are credited and that the original publication in this journal is cited, in accordance with accepted academic practice. No use, distribution or reproduction is permitted which does not comply with these terms. 